PRAMANA

- journal of

physics (c) Indian Academy of Sciences

Vol. 55, Nos 5 \& 6

Nov. \& Dec. 2000

pp. $645-654$

\title{
Space and astrophysical plasmas: Pervasive problems
}

\author{
CHANCHAL UBEROI \\ Department of Mathematics, Indian Institute of Science, Bangalore 560 012, India
}

\begin{abstract}
The observations and measurements given by Earth orbiting satellites, deep space probes, sub-orbital systems and orbiting astronomical observatories point out that there are important physical processes which are responsible for a wide variety of phenomena in solar-terrestrial, solar-system and astrophysical plasmas. In this review these topics are exemplified both from an observational and a theoretical point of view.
\end{abstract}

Keywords. Heliosphere; solar corona; Alfven resonances; magnetic reconnection.

PACS Nos 95.30 Q; 96.50 R

\section{Introduction}

Space science is generally defined as investigations that are conducted by scientists using earth orbiting satellites, deep space probes like Pioneer series and sub-orbital systems. Space plasma sciences are now a very highly developed multidisciplinary area of research and can be divided into three areas: solar-terrestrial or Sun-Earth relations, solar-systems exploration and astrophysics.

Though space science grew out of the rocket research which followed the end of World War II, the early investigations which began in 1946 concentrated on studies of the Earth's atmosphere, solar radiation and cosmic rays from space. It was in 1958 that launch of Explorer I and subsequent discovery of Van Allen belts which brought the scientific research in space sciences on to the list of other branches of physical and astrophysical sciences. These earlier flights of Pioneer I and subsequent series which laid the foundation of area of space plasma physics which as we now know has become a highly developed multidisciplinary area of research to understand the Sun-Earth or solar-terrestrial relations.

In 1960 with launch of later in series of Pioneer 5, we moved away from the Earth into the interplanetary space towards the planets. Pioneer 10 and 11, Mariner 10, Viking and then Voyager 1 and 2 all equipped with sophiosticated instruments in 1977 brought us the dramatic results of the space plasmas in the neighbourhood of outer planets, Jupiter, Saturn and then Uranus and Neptune. The Sun from space was also studied during this period by Orbiting Solar Observatory (OSO-I). This gave us ample data for the solar-system plasmas.

In addition to Sun-Earth relations and solar systems exploration a new space science discipline astrophysics or space astronomy (as it is sometimes called as it is a mixture of ancient astronomy, modern physics and high technology of space experimentation) came 
up. X-ray and ultraviolet astronomy and now $\gamma$-ray astronomy interests led to Orbiting Astronomical Observatories.

The study of science of space plasmas is truly an interdisciplinary field of research with scientific interest that does not lend itself to simple arbitrary boundaries. For example, the sun as a star is of interest to astrophysics, while the Sun as a source of radiation is of importance in understanding Sun-Earth relationships. The studies of particles and fields is essential to understanding the Sun, the Earth, the planets, and stars and the many enigmatic objects in the universe. In this review talk therefore I decided to point out and discuss some universal physical plasma processes which are responsible for a wide variety of astrophysical, solar and magnetospheric plasma phenomena. The plasma processes which I have considered are magnetic reconnection and associated phenomena of tearing mode instability, Kelvin-Helmholtz (K-H) instability and surface wave resonant interaction.

In recent years the Earth-orbiting satellites have provided a wealth of details in agreement with magnetic reconnection models from in-situ measurements of reconnection processes in natural plasma. Because the satellite measurements have so much to say about the physics of these processes, they should be of interest not only to magnetospheric physicists but to astrophysicists and solar physicists as well. The aim of this talk is to discuss these pervasive problems for Earths' magnetosphere, solar flares, coronal heating, heliosphere, galactic halo, prominences and some other examples.

Earths' immediate space environment is driven by the energy contained in the solar wind plasma and this is controlled by magnetic reconnection occurring on the interface between the solar wind and the magnetospheric plasmas. A large scale electric field is imposed across the northern and southern polar regions of the ionosphere. Electric currents drive large-scale plasma convection, particle energization, and the aurora. The opening of the Earth's magnetic field across the magnetopause allows plasma to flow into and out of the magnetosphere, which provides an important diagnostic tool from which we can infer field line topology, reconnection rate, energy transfer and so on (figure 1). The Earths' space environment therefore acts as a laboratory for reconnection. The in-situ measurements have a wealth of data to understand this process.

\section{Earth's magnetosphere}

The magnetopause acts like a boundary between the interplanetary plasma and the magnetospheric plasma. Existence of surface waves along the magnetopause and the dynamics of these to understand ultra low-frequency (ULF) waves or micropulsations was first suggested by Dungey in 1955 [1]. With advent of space age now we have ample spacecraft observations of surface waves and oscillations of the Earth's magnetospheric boundary.

\subsection{Spacecraft observations}

Multi-spacecraft ISEE mission [2] has given detailed observations of surface waves with periods of about $2 \mathrm{~min}$ or more along the magnetopause. It was also seen that magnetopause is more oscillatory for southward interplanetary magnetic field (IMF) than for northward IMF. However evidence of magnetic reconnection at the magnetopause come 

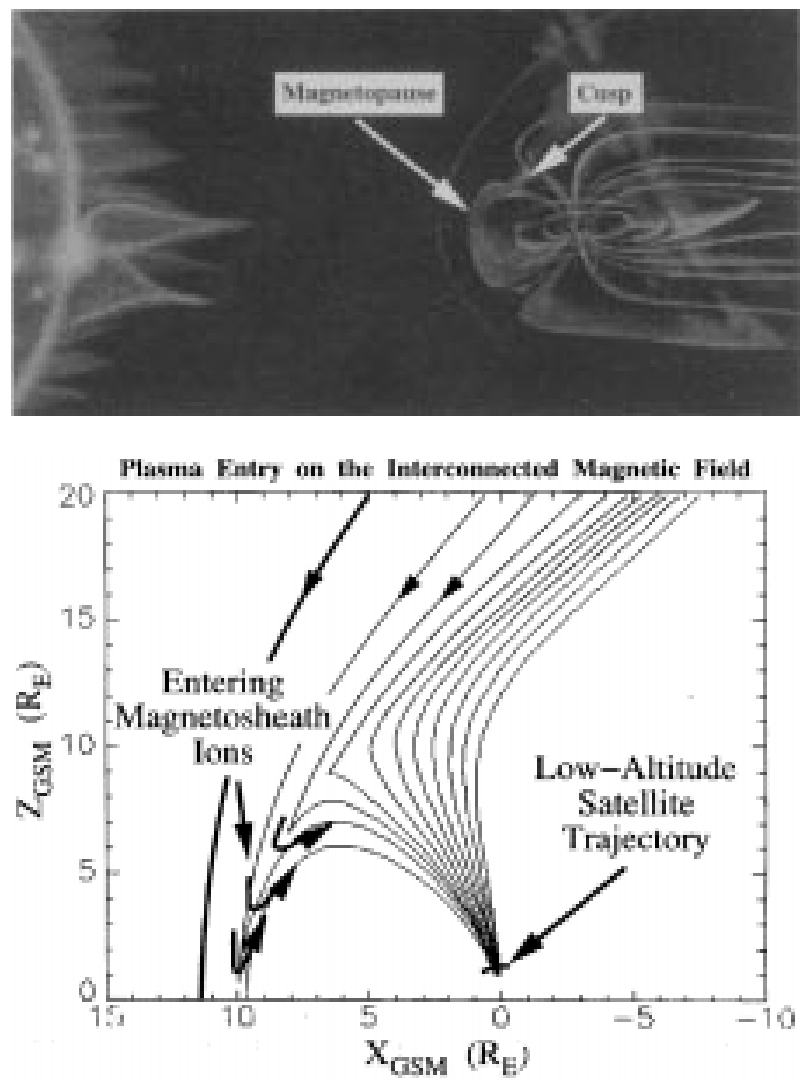

Figure 1. The upper illustration shows the solar wind-magnetosphere system with magnetopause, the boundary separating the Earth's magnetic field from the magnetic field in interplanetary space. The lower diagram illustrates the interaction of the Earth's magnetic field to the interplanetary field resulting from reconnection. The ions outside the magnetopause can freely enter the magnetosphere on the interconnected field lines and will then be detected by a low altitude satellite (from the article by T G Onsager and R C Elphic, EOS, June 1996).

with the recent ISEE observations (figure 2) that of magnetic flux tubes of cross section $1 R_{E}$ near Earth's magnetosphere which were isolated from each other. It was identified that the observed $B_{n}$ polarity reverses with the passing of a localized reconnected flux tube. These were called as flux transfer events (FTEs), as these events seem to be responsible for flux or momentum transfer into the magnetosphere during the solar-wind geomagnetic field interaction. FTEs are characterized by prominent isolated bipolar (+ve then -ve or vice versa) perturbation in the component normal to the magnetopause. They occur intermittently every 5 to 15 minutes. Recent survey confirms that onset of FTE's is controlled by southward component of IMF. It is inferred from observations (figure 3 ) that flux transferring reconnection does not occur unless the magnetic field at the magnetopause has been southward for the order of $7 \mathrm{~min}$ or more. This time delay is real and not due to spacecraft not being in the right location at the right time to get the observations correct. From obser- 
vations it is noted additionally that there is no apparent solar wind or other interplanetary driver for the observed initial delay of the first FTE [3].
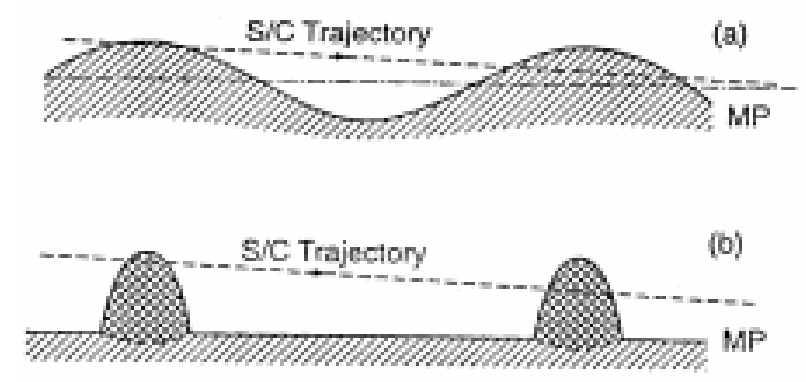

Figure 2. (a) A spacecraft passes a wavy magnetopause. (b) A spacecraft passes FTEs (double-hatched region) or surface wave pulses (thick solid line).

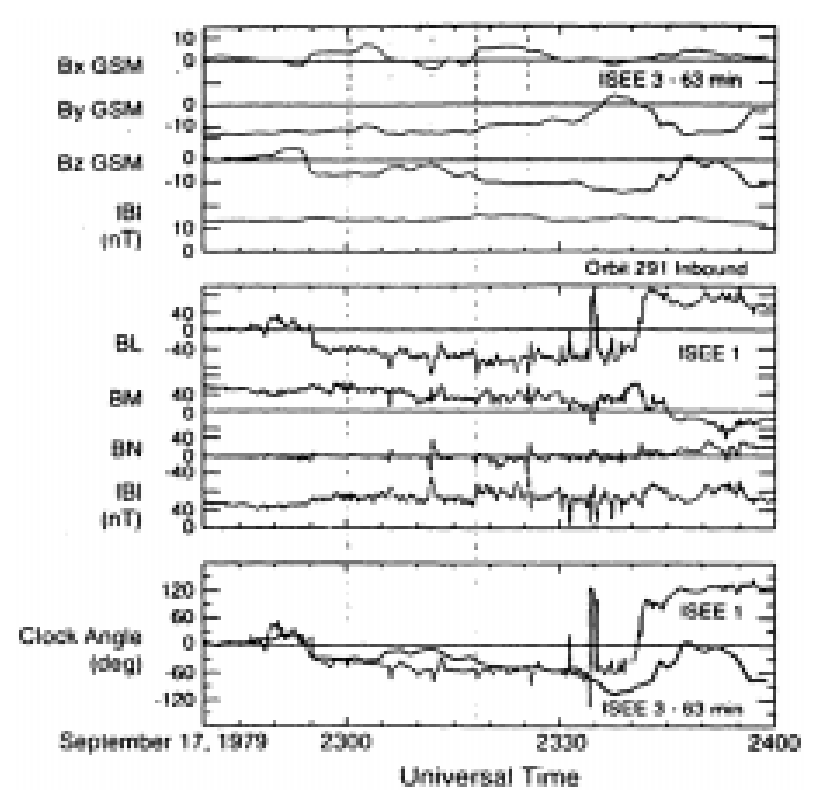

Figure 3. Flux transfer events detected by ISEE-1 in the magnetosheath under conditions of approximately steady IMF conditions. The top four traces show the IMF at ISEE-3 measured in GSM coordinates. The middle four traces show the magnetic field at ISEE-1 measured in boundary normal co-ordinates and the botom panel shows the clock angle measured by ISEE-1 and 3 that was used to determine time delay of the two observations (from Russel et al, 1997). Note: ISEE3 detects nearly constant southward IMF and the first FTE is seen 5 minutes after the southward turning is detected at ISEE1. 


\subsection{Mathematical models}

There are different mathematical models for FTE's. Tearing instability is an integral part of all the models. Fu and Lee [4] gave the multiple-line reconnection model. But the assumption of perpendicular velocity flow in that model may not be always correct. In absence of such a field Liu and Hu [5] suggested vortex induced magnetic reconnection (VIMR). In this model it was suggested that tearing instability is induced by the vortices formed due to excitation of the KH instability. The analysis was non-linear and could explain the large scale magnetic islands but Chen et al [6] showed that linear interaction between K-H and tearing instability is not possible. Vortex formation requires $V>V_{c}$, the threshold velocity of the K-H instability. This condition excludes the subsolar magnetopause as the velocity $V$ being nearly zero there. Vortices should be almost fully developed otherwise the reconnection flux is small. During their formation the flow vortices move down stream at a speed close to the magnetosheath plasma velocity. So vortex would have moved more than $2 R_{E}$, thus excluding the major portion of the dayside magnetopause as a possible location to observe FTEs.

The surface induced magnetic reconnection model (SWIMR) given by Uberoi [7] has its origin in the difference of resonant behaviour of surface Alfven waves at the zero and non-zero singular points, as the role played by surface waves at these points differ. At the neutral point, the zero frequency surface waves (which are symmetric mode of a plasma layer) couple to the low frequency end of the Alfven continuum. When the resistivity is switched on, these surface modes couple with the tearing mode instability of the layer. Uberoi et al [8] have explained the observations of FTE's at magnetopause by SWIMR. Dimensions of islands formed and intrinsic time scale for the reconnection to start [9] agreed well with observed values (figure 4).

\section{Solar cornal heating}

It is generally agreed that the source of energy by which the solar corona is heated to temperature more than 100 times the temperature at photosphere level is ultimately the convective motion of the very dense plasma in the photosphere. However, it is still uncertain what the mechanisms are by which that energy is transmitted to the corona and then dissipated to provide the necessary heating. Certainly the magnetic field is responsible for the transmission of energy by coupling the photosphere dynamically to the corona.

\subsection{Satellite observations}

Solar corona is highly structured and inhomogeneous, containing many discrete loops of varying sizes and UV observations indicate the presence of MHD waves propagating in the coronal plasmas.

In the previous solar maximum a balloon borne hard X-ray observation with very high sensitivity discovered microflares with non-thermal hard X-ray spectra. This discovery has led to the speculation that numerous microflares or nanoflares can be possible source of heating the corona. 

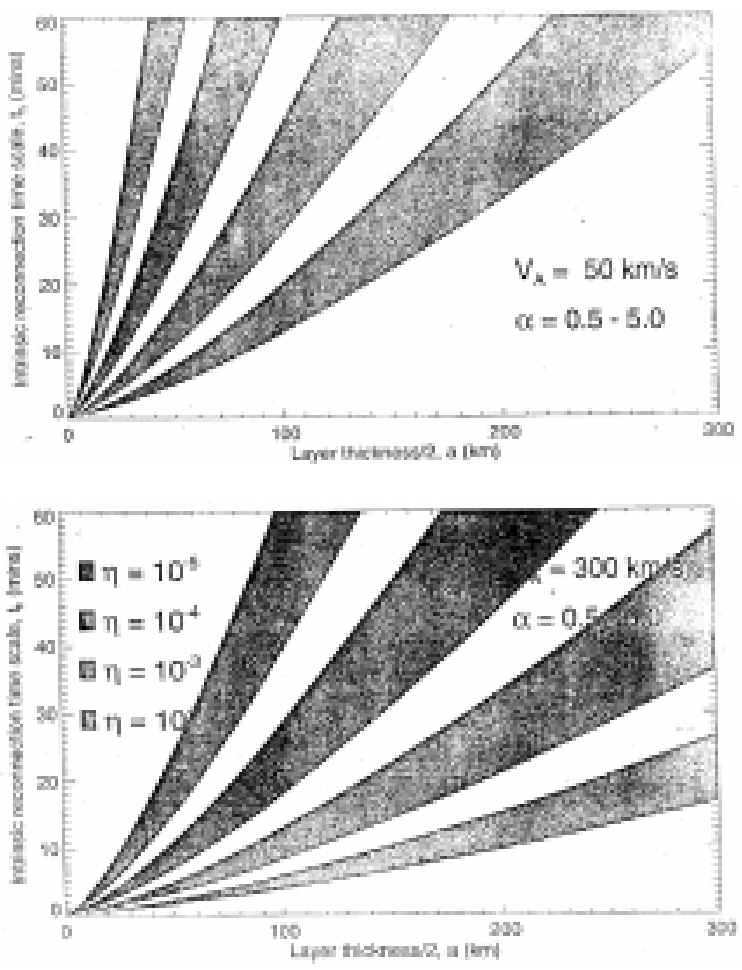

Figure 4. Variation of $t_{h}$, the intrinsic timescale for the onset of reconnection (min.) with a $(\mathrm{km})$, the half-thickness of the neutral layer, for different values of the resistivity $\eta$ ranging from $10^{-2}$ to $10^{-5} \mathrm{ohm} \mathrm{km}$ for two values of Alfven velocity $V_{A}(\mathrm{~km} / \mathrm{s}) . \alpha$ represents the degree of ionization (from Uberoi et al [9]).

Recent satellite observations made by soft X-ray telescope about the Yohkoh and SOHO satellites reveals that active regions show many small flare-like brightenings. It was suggested that transient brightenings revealed by soft X-ray telescope are the soft X-ray counterpart of the hard X-ray microflares. The observed X-ray jets and $H \alpha$ surges are associated with microflares (figure 5). Studying the morphology of transient brightenings (142 observed transient brightenings) Shimizu et al [11] showed that most of these brightenings are due to magnetic interaction of multiple loops. X-ray emission from the footpoints in the early phase suggests that the hot plasma in the brightening loops comes from chromospheric matter or low temperature matter present around the base of the coronal loops prior to the brightening. Enhanced X-ray emission at the contact points are due to local plasma heating by magnetic interaction.

\subsection{Mathematical models}

The above observation suggest the following models for dissipative processes in coronal heating. 

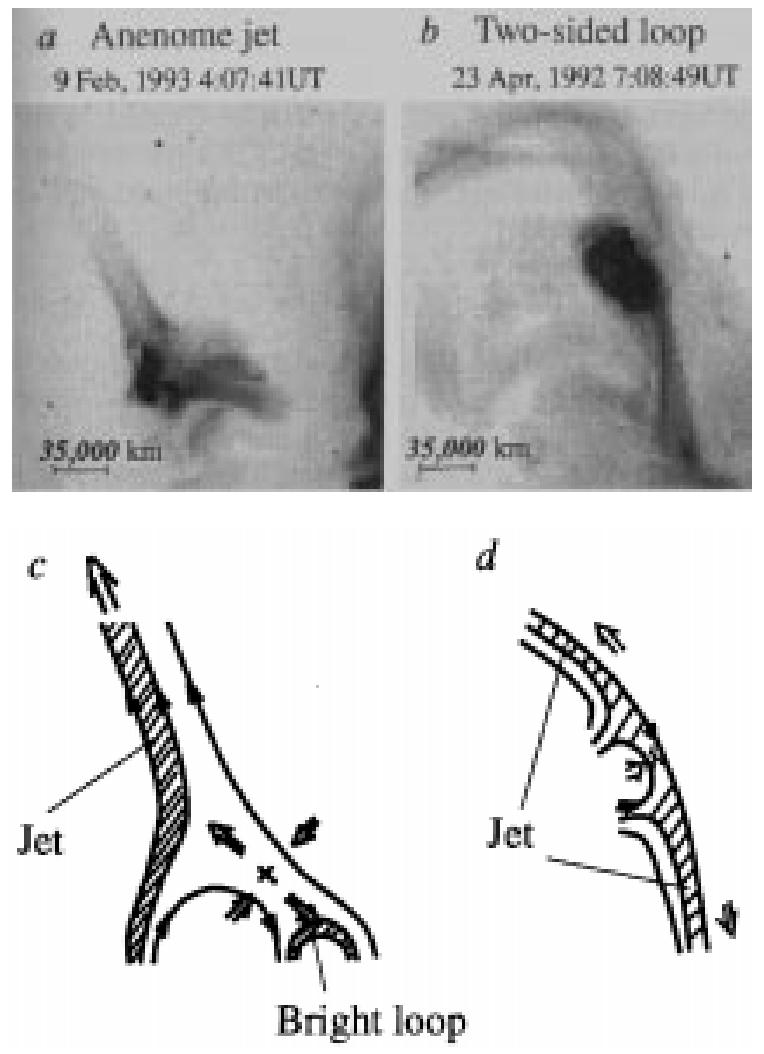

Figure 5. Examples of two types of solar coronal X-ray jets (a and $\mathbf{b}$ ) observed by the Yohkoh soft X-ray telescope. $\mathbf{c}$ and $\mathbf{d}$ are schematic pictures of models for each type (for references see [10]).

1. Alfven resonant absorption, a high frequency phenomena that appears to work well with coronal loop heating (for example see [12]).

2. Plasmoid-induced reconnection model given by Shibata [13] for microflares (figure 6).

3. Forced or driven magnetic reconnection [14].

It has been noted by Uberoi and Zweibel [15] that this mechanism is embedded in the Alfven resonant theory.

The surface wave induced magnetic reconnection model (SWIMR) is presently being discussed by Pandey et al [16]. The advantage with this model is that it shows that both the first and third mechanism above are essentially the manifestation of one and the same physical process - namely the excitation of the MHD surface waves spectrum. The energy from the high frequency part of the MHD spectra is absorbed in the coronal plasma by resonantly exciting the kinetic Alfven waves, the low frequency part of the spectra excites the low frequency resistive modes in the system which leads to magnetic reconnection. 

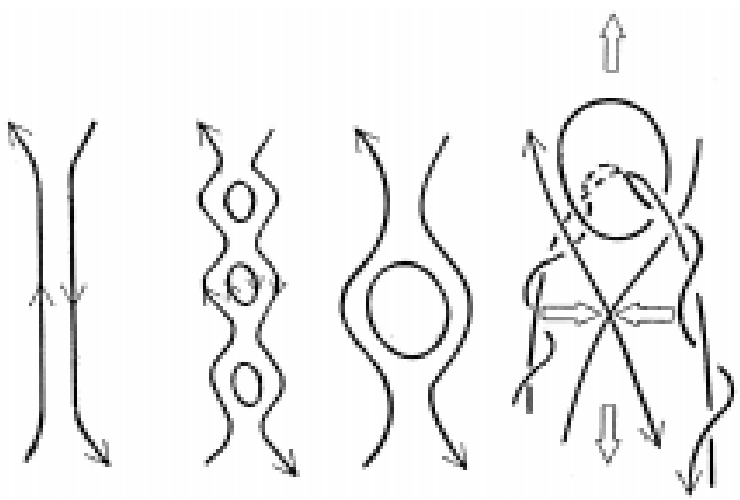

Figure 6. Schematic drawings showing current sheet formation, tearing, coalescena, plasmoid ejection in the plasma-induced magnetic reconnection model [13].

\section{Heliosphere}

At present, there is no doubt that the local interstellar medium (LISM) is a partially ionized gas. Therefore models of the interaction of the solar wind with interstellar medium must take into account the presence of the LISM hydrogen atoms (figure 7). Heliosphere, the region of influence of solar wind, is immersed in partially ionized plasma referred to as the very local interstellar medium (VLISM). The VLISM flows at about $25 \mathrm{~km} / \mathrm{s} \mathrm{has} \mathrm{a}$ temperature of about $20^{\circ} \mathrm{K}$ with plasma density in the range $0.05-0.4 \mathrm{~cm}^{-3}$ [17]. Plasma waves in the solar wind within $1 \mathrm{AU}$ from the Sun and near the ecliptic plane are well known. But now Ulysses observations cover a range of 0.2 to $448 \mathrm{~Hz}$ in solar wind at distance 1 to $5 \mathrm{AU}$ from the Sun. Heliospheric current sheets (HCS) are also detected by magnetometer experiments on Ulysses. The maximum northern latitude reached by the HCS by Ulysses on 30 September 1996 was $25.5^{\circ}$ N. From WIND observation, surface waves of a few thousand kilometers has been reported. All these facts emphasize the importance of study of SWIMR in partially ionized plasma.

From the dispersion relation [18] for the surface waves along a partially ionized plasmaplasma interface of finite thickness with an equilibrium magnetic field in the plane of the interface, it can be seen that it can support two types of surface modes of oscillations, namely symmetric and asymmetric. In the low frequency mode thay have different characteristics than that of fully ionized plasma (see figure 8 for example). For a neutral layer, it is the symmetric mode that has zero frequency, whereas the asymmetric mode has a finite frequency at the neutral layer. Can near zero frequency surface waves with $k a<<1$ exist along heliopause? For heliopause magnetic field is $2 \mu \mathrm{G}$ (microgauss) and $a \simeq 10^{3} \mathrm{~km}$, ratio of neutral and ion density being $10^{2}$, one finds frequencies of order $10^{-13} \mathrm{~Hz}$ (for $k a=10^{-3}$ ). So near zero frequency surface waves can exist along heliopause. It is interesting that there is a cutoff thickness $a_{c}=\frac{V_{A 1}(k a)}{2 \nu_{n i}}\left(1+\alpha_{1}\right)^{1 / 2}$, for layer with thickness less than $2 a_{c}$ surface wave will not exist. Here $V_{A 1}$ is the Alfven velocity in inner region of plasma layer, $k$ is the wave number, $a$ is the thickness of the layer, $\nu_{n i}$ is neutral ion collision frequency and $\alpha_{1}=\left(\nu_{i n} / \nu_{n i}\right)$ in the inner region. The condition $a_{c}<a$ is satisfied for heliopause for high collisional frequency. 


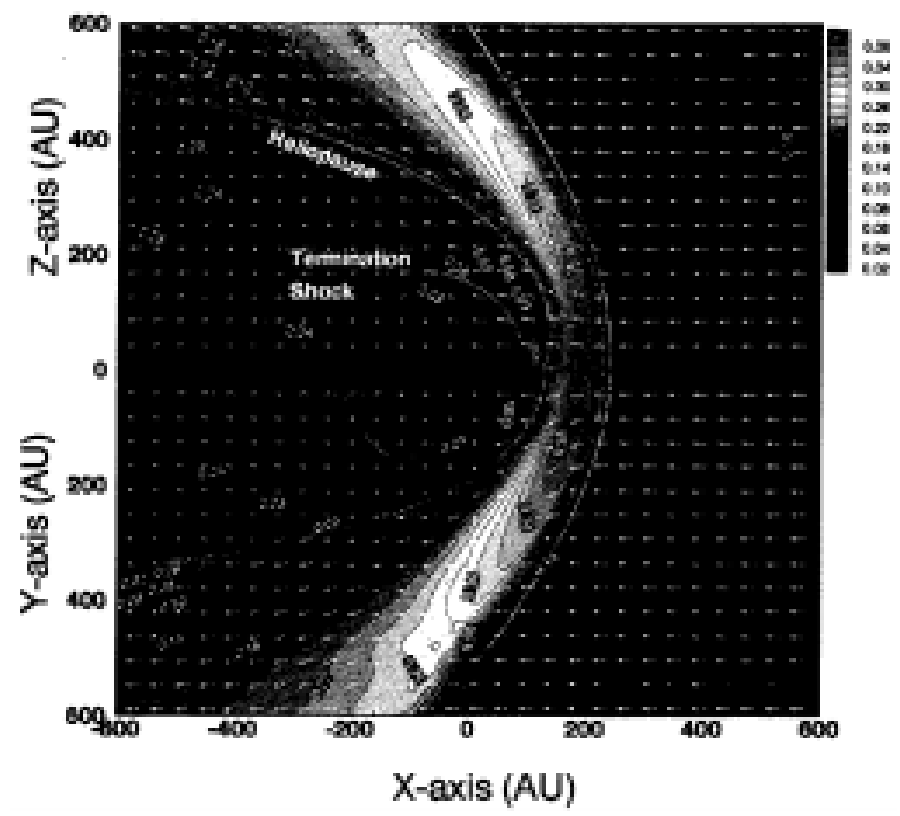

Figure 7. Interstellar neutral hydrogen density $\left(\mathrm{cm}^{-3}\right)$ (contour and shades) and normalized flow vectors as a function of distance in the polar plane (top panel) and in the ecliptic plane (bottom panel). The positions of the plasma boundaries are indicated (from Paul and Zank [17]).

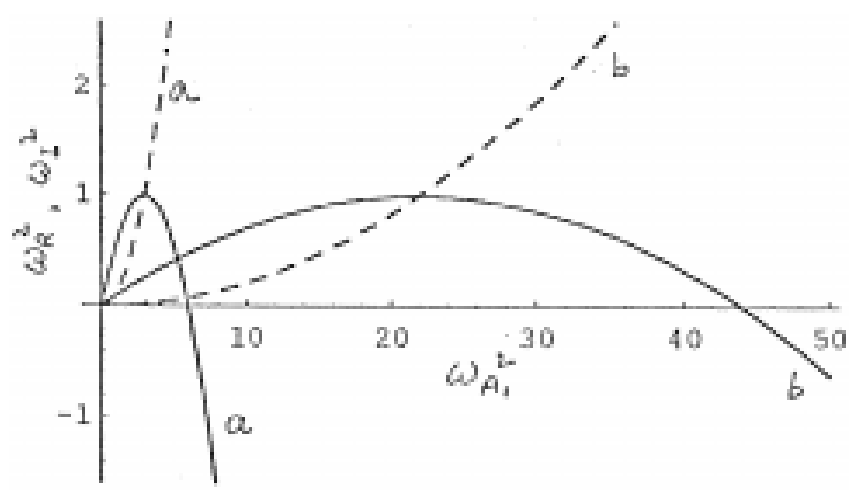

Figure 8. As an example the dispersion curve showing variation of real $w_{R}$ (solid lines) and imaginary part of the frequency $\omega_{I}$ (dotted lines) with Alfven wave frequency in medium $1, \omega_{A 1}^{2}$ for the strongly coupled symmetric surface mode propagating along the plasma-plasma interface bounded by, medium 1 and 2 (a) $\alpha_{1}=0.5$ (b) $\alpha_{1}=10, \alpha_{1}$ represents the degree of ionization in medium 1 (from Uberoi and Datta [18]).

From the above discussion it can be said that SWIMR is a possible mechanism of reconnection along the interface of heliopause local interstellar medium. 


\section{Conclusion}

The SWIMR model for partially ionized plasma systems can be extended to understand the energetics in the galactic halo by considering a model interaction of a high velocity cloud with the galactic disc [19]. The solar prominences with strongly sheared magnetic fields and molecular clouds are other examples where reconnection process is strongly effected by the ion-neutral couplings and therefore can be studied with SWIMR model [20]. The problem of theoretical understanding of Alfven resonant phenomena, tearing instability and magnetic reconnection therefore is seen to be prevading various areas of space and astrophysical plasmas.

\section{References}

[1] J W Dungey, Electrodynamics of the outer atmosphere, Proc. Conference Phys. Soc. London (1955)

[2] P Song, R C Elphic and C T Russell, Geophys. Res. Lett. 15, 744 (1988)

[3] C T Russel, Le, H Kawano, S M Petninec and T I Zhang, Adv. Space Res. 19, 1913 (1997)

[4] Z F Fu and L C Lee, Geophys. Res. Lett. 12, 291 (1985)

[5] Z X Liu and Y D Hu, Geophys. Res. Lett. 15, 752 (1988)

[6] Q Chen, A Otto and L C Lee, J. Geophys. Res. 102, 181 (1997)

[7] C Uberoi, J. Plasma Phys. 52, 215 (1994)

[8] C Uberoi, L J Lanzerotti and A Wolfe, J. Geophys. Res. 101, 24979 (1996)

[9] C Uberoi, L J Lanzerotti and C G Maclennan, J. Geophys. Res. 104, 25, 153 (1999)

[10] T Yokoyama and K Shibata, Nature 375, 42 (1995)

[11] T Shimizu, S Tsuneta, L W Acton, J R Lemen, O Yoshiaki and Y Uchida, Astrophys. J. 422, 906 (1994)

[12] J V Hollweg and G Yang, J. Geophys. Res. 93, 5423 (1988)

[13] K Shibata, Observational Plasma Astrophysics: Five years of Yokoh and Beyond (Kluwer Academic Pub., Netherlands, 1998) pp. 187-196

[14] X Wang and A Bhattacharjee, Astrophys. J. 401, 371 (1992)

[15] C Uberoi and E Zweibel, J. Plasma Phys. 62, 345 (1999)

[16] B P Pandey, C Uberoi and G S Lakhina (to be published)

[17] H L Pauls and G P Zank, J. Geophys. Res. 102, 19, 779 (1997)

[18] C Uberoi and A Datta, Phys. Plasmas (to be published) See also C Uberoi and A Datta, Phys. Plasmas 5, 4149 (1998)

[19] G T Birk, H Lesch and F Zimmer, Phys. Scr. T74, 89 (1998)

[20] E Zweibel, Astrophys. J. 340, 550 (1989) 